\title{
Vacuum Polarization of Massless Spinor Field in Global Monopole Spacetime
}

\author{
E.R. Bezerra de Mello *, V.B. Bezerra ${ }^{\dagger}$, and N.R. Khusnutdinov ${ }^{\ddagger}$ \\ Departamento de Física, Universidade Federal da Paraíba, \\ Caixa Postal 5008, CEP 58051-970 João Pessoa, Pb, Brazil
}

(October 13, 2018)

\begin{abstract}
We calculate the renormalized vacuum average of the energy-momentum tensor of massless lefthanded spinor field in the pointlike global monopole spacetime using point-separation approach. The general structure of the vacuum average of the energy-momentum tensor is obtained and expressed in terms of $\left\langle T_{0}^{0}\right\rangle^{r e n}$ component, explicit form of which is analyzed in great details for arbitrary solid angle deficit.
\end{abstract}

98.80.Cq, 14.80.Hv, 95.30.S

\section{INTRODUCTION}

It is well-known that different types of topological objects may have been created by vacuum phase transition in the early Universe [1,2]. These include domain walls, cosmic strings and monopoles. Among them, cosmic strings and monopoles seem to be the best candidates to be observed.

Global monopole is a heavy object formed in the phase transition of a system composed of a self-coupling scalar field triplet $\phi^{a}$ whose original global $O(3)$ symmetry is spontaneously broken to $U(1)$. The scalar matter field plays the role of an order parameter which outside the monopole core acquires a nonvanishing value. The main part of the monopole's energy is concentrated into its small core. The simplest model which gives rise to a global monopole is described by the Langrangian density below, and was analysed by Barriola and Vilenkin [3]

$$
L=\frac{1}{2}\left(\partial_{\mu} \phi^{a}\right)\left(\partial^{\mu} \phi^{a}\right)-\frac{1}{4} \lambda\left(\phi^{a} \phi^{a}-\eta^{2}\right)^{2},
$$

Coupling this matter field with the Einstein equations, a spherically symmetric metric tensor given by the line element

$$
d s^{2}=-B(r) d t^{2}+A(r) d r^{2}+r^{2}\left(d \theta^{2}+\sin ^{2} \theta d \varphi^{2}\right),
$$

presents solutions for the functions $B(r)$ and $A(r)$ far from the monopole's core given by

$$
B=A^{-1}=1-8 \pi \eta^{2}-2 M / r .
$$

The mass parameter $M \sim M_{\text {core }}$. Numerical details concerning with this function can be seen in Ref. [4]. Neglecting the mass term we get the pointlike global monopole spacetime with metric

$$
d s^{2}=-\alpha^{2} d t^{2}+d r^{2} / \alpha^{2}+r^{2}\left(d \theta^{2}+\sin ^{2} \theta d \varphi^{2}\right),
$$

where the parameter $\alpha^{2}=1-8 \pi \eta^{2}$. The energy momentum tensor of this monopole has diagonal form and reads: $T_{t}^{t}=T_{r}^{r}=\left(\alpha^{2}-1\right) / r^{2}$ and $T_{\theta}^{\theta}=T_{\varphi}^{\varphi}=0$. The main peculiarity of this space is a solid angle deficit which is the difference between the solid angle in the flat spacetime $4 \pi$ and the solid angle in the global monopole spacetime which is given by $4 \pi \alpha^{2}$. For $\alpha<1$ one has solid angle deficit and for $\alpha>1$ one has the solid angle excess. (We would like to call attention that the physical values for $\alpha$ predicted by field theory is smaller than unit D). The spacetime produced by a global monopole has no Newtonian gravitational potential in spite of the geometry produced by this heavy object has a non-vanishing curvature. For this reason the mass of the monopole is divergent and proportional to the distance from monopole origin [3]. In context of the monopole formation the cosmological horizon is a natural cutoff distance for the monopole's mass.

\footnotetext{
*e-mail: emello@fisica.ufpb.br

†e-mail: valdir@fisica.ufpb.br

${ }^{\ddagger}$ On leave from Kazan State Pedagogical University, Kazan, Russia; e-mail: nail@dtp.ksu.ras.ru

${ }^{1}$ In fact for a typical grand unified theory the parameter $\eta$ is of order $10^{16} \mathrm{Gev}$. So $1-\alpha^{2}=8 \pi \eta^{2} \sim 10^{-5}$
} 
Although the global monopole has no Newtonian gravitational potential it gives enormous tidal acceleration $a \sim 1 / r^{2}$ which is important from the cosmological point of view and may be used for obtaining upper bound on the number density of them in the Universe, which is at most one global monopole in the local group galaxies [5]. However, the numerical simulations made by Bennet and Rhie show that real upper boundary is smaller than that given in [5] by many orders [6]. In fact, one has scaling solution with a few global monopoles per horizon volume [6].

The quantum effects due to the monopole background in the matter fields have been considered explicitly for scalar field in Ref. [7] and by general consideration in Ref. 8]. It has been shown from general consideration that the vacuum expectation value of the energy-momentum tensor of massless fields has the following general form

$$
<T_{\mu}^{\nu}>^{r e n}=\frac{S_{\mu}^{\nu}(\mu r)}{r^{4}}
$$

where the tensor $S_{\mu}^{\nu}$ depends on the arbitrary mass scale parameter $\mu$ and the metric coefficient $\alpha$. In Ref. [8], it has been assumed that the tensor $S_{\mu}^{\nu}$ is the function of the metric parameter $\alpha$, only. Manifest calculations in scalar case have shown [7] that this tensor depends on renormalization mass parameter $\mu$ and it has the following structure $S_{\mu}^{\nu}=A_{\mu}^{\nu}+B_{\mu}^{\nu} \ln \mu r$, where tensors $A_{\mu}^{\nu}$ and $B_{\mu}^{\nu}$ depend only on the $\alpha$. This is in agreement with Wald [9] who noted that an unambiguous prescripton for $\left\langle T_{\mu}^{\nu}>^{r e n}\right.$ cannot be defined without introducing a length scale. Nevertheless, one-loop Einstein equations do not depend on scale parameter $\mu$ due to renormalization group equation. Back reaction problem in the scalar massless case has been investigated by Mazzitelli and Lousto [f]. It must be noted that only the general structure of vacuum expectation value of the energy-momentum tensor has been considered. There, it was not obtained an explicit form of the tensor $S_{\mu}^{\nu}$.

In this paper we would like to obtain the explicit value of this tensor, considering the massless spinor field on the background of the pointlike global monopole with metric given by Eq.(1). As opposed to the massless scalar field case [7] we get a simpler expression for the Green function and the energy-momentum tensor which is obtained in closed form, for arbitrary angle deficit.

The analysis of the quantum behavior of two massless left-handed $S U(2)$ doublets fermionic field in the background of a pointlike monopole, taking into account the magnetic field, has been developed by Ren [10]. There, the Rubakov and Callan effect was analysed, and found that there appears a small correction due to the parameter $\alpha$ into the fermion number condensate.

This paper is organized as follows. In Sec. [1] we consider the Green functions for massive and massless spinor fields on the background of the pointlike global monopole and obtain, in closed form, the Euclidean Green function for massless left-handed spinor field. In Sec. III] we analyze this function at coincidence limit and extract all divergencies from it in manifest form. We obtain also the general structure of renormalized energy-momentum tensor. Each component of this tensor may be expressed in terms of only the zero-zero component $<T_{0}^{0}>^{\text {ren }}$ which is analyzed with great details. In Sec. IV we summarize our results. Appendices A and B contain some technical formulas. The signature of the spacetime, the sign of Riemann and Ricci tensors are the same as in Christensen paper [17]. We use units $\hbar=c=G=1$.

\section{SPINOR GREEN FUNCTIONS}

In this section we want to obtain the expression for the fermion propagator of massive spinor field in the pointlike monopole spacetime. Massless field will be recognized as particular case. The spinor Feynman propagator is defined as follows [1]

$$
i \mathcal{S}_{\mathcal{F}}\left(x, x^{\prime}\right)=<0\left|T\left(\Psi(x) \bar{\Psi}\left(x^{\prime}\right)\right)\right| 0>,
$$

where $\bar{\Psi}=\Psi^{+} \gamma^{0}$ which, under the Lorentz transformation $\Psi \rightarrow S(\triangle) \Psi$, transforms as $\bar{\Psi} \rightarrow \bar{\Psi} S^{-1}(\triangle)$, where $\triangle$ is the parameter of the transformation, and $S(\triangle)$ is a local representation of the Lorentz group.

This propagator obeys the following differential equation

$$
(i \not \nabla-M) \mathcal{S}_{\mathcal{F}}\left(x, x^{\prime}\right)=\frac{1}{\sqrt{-g}} \delta^{(4)}\left(x-x^{\prime}\right) I_{4},
$$

where $g=\operatorname{det}\left(g_{\mu \nu}\right)$. The covariant derivative operator in the above equation is

$$
\not \nabla=e_{(a)}^{\mu} \gamma^{(a)}\left(\partial_{\mu}+\Gamma_{\mu}\right)
$$

$e_{(a)}^{\mu}$ being the vierbein satisfying the condition 


$$
e_{(a)}^{\mu} e_{(b)}^{\nu} \eta^{a b}=g^{\mu \nu}
$$

and $\Gamma_{\mu}$ is the spin connection, given in terms of the flat spacetime $\gamma$-matrix by

$$
\Gamma_{\mu}=-\frac{1}{4} \gamma^{(a)} \gamma^{(b)} e_{(a)}^{\nu} e_{(b) \nu ; \mu}
$$

The Green function given in (7) is a bispinor, i.e. it transforms as $\Psi$ at $x$ and as $\bar{\Psi}$ at $x^{\prime}$.

If a bispinor $\mathcal{D}_{\mathcal{F}}\left(x, x^{\prime}\right)$ satisfies the differential equation below

$$
\left(\square-M^{2}-\frac{1}{4} R\right) \mathcal{D}_{\mathcal{F}}\left(x, x^{\prime}\right)=-\frac{1}{\sqrt{-g}} \delta^{(4)}\left(x-x^{\prime}\right) I_{4},
$$

where the generalized d'Alembertian is expressed by

$$
\square=g^{\mu \nu} \nabla_{\mu} \nabla_{\nu}=g^{\mu \nu}\left(\partial_{\mu} \nabla_{\nu}+\Gamma_{\mu} \nabla_{\nu}-\Gamma_{\mu \nu}^{\alpha} \nabla_{\alpha}\right)
$$

then the spinor Feynman propagator may be read as

$$
\mathcal{S}_{\mathcal{F}}\left(x, x^{\prime}\right)=(i \not \nabla+M) \mathcal{D}_{\mathcal{F}}\left(x, x^{\prime}\right)
$$

which shows that the non-minimal coupling to the curvature does play a role when spinor fields are considered.

Now after this brief review about the calculation of spinor Feynman propagator in the general manifold, let us specialize it to the spacetime of a global monopole. We shall choose the following basis tetrad:

$$
e_{(a)}^{\mu}=\left(\begin{array}{cccc}
1 / \alpha & 0 & 0 & 0 \\
0 & \alpha \sin \theta \cos \varphi & \cos \theta \cos \varphi / r & -\sin \varphi / r \sin \theta \\
0 & \alpha \sin \theta \sin \varphi & \cos \theta \sin \varphi / r & \cos \varphi / r \sin \theta \\
0 & \alpha \cos \theta & -\sin \theta / r & 0
\end{array}\right)
$$

For this case, the only non-zero spin connections are

$$
\begin{aligned}
& \Gamma_{2}=\frac{1-\alpha}{2}\left[\gamma^{(1)} \gamma^{(2)} \cos \varphi+\gamma^{(2)} \gamma^{(3)} \sin \varphi\right] \\
& \Gamma_{3}=-\frac{1-\alpha}{2}\left[\gamma^{(1)} \gamma^{(2)} \sin \theta+\gamma^{(1)} \gamma^{(3)} \cos \theta \sin \varphi-\gamma^{(2)} \gamma^{(3)} \cos \theta \cos \varphi\right] \sin \theta
\end{aligned}
$$

If we had made another choice for the vierbein, for example $e_{(a)}^{\mu}=\operatorname{diag}(1 / \alpha, \alpha, 1 / r, 1 / r \sin \theta)$, the only non-zero spin connection would be $\Gamma_{2}=-\alpha \gamma^{(1)} \gamma^{(2)} / 2$ and $\Gamma_{3}=-\alpha\left[\gamma^{(1)} \gamma^{(3)} \sin \theta+\gamma^{(2)} \gamma^{(3)} \cos \theta\right] / 2$. Although this tetradic basis is simpler than previous one given in (14), the spin connection obtained by the former does not vanish when we take Minkowski limit $\alpha=1$.

In order to obtain the explicit form for the differential equation for the bispinor $\mathcal{D}_{\mathcal{F}}$ given in (11) in this geometry we shall adopt the following representation for the $\gamma$-matrix:

$$
\gamma^{(0)}=\left(\begin{array}{cc}
1 & 0 \\
0 & -1
\end{array}\right), \gamma^{(k)}=\left(\begin{array}{cc}
0 & \sigma^{k} \\
-\sigma^{k} & 0
\end{array}\right)
$$

$\sigma^{k}$ being the $2 \times 2$ Pauli matrices. These matrices above obey the anticommutator relations $\left\{\gamma^{(a)}, \gamma^{(b)}\right\}=-2 \eta^{a b}$.

After some intermediate steps, we get the following expression for the d'Alembertian operator

$$
\square=-\frac{1}{\alpha^{2}} \partial_{t}^{2}+\frac{\alpha^{2}}{r^{2}} \partial_{r}\left(r^{2} \partial_{r}\right)-\frac{1}{r^{2}} \vec{L}^{2}-\frac{(1-\alpha)^{2}}{2 r^{2}}-\frac{1-\alpha}{r^{2}} \vec{\Sigma} \cdot \vec{L},
$$

where

$$
\vec{\Sigma}=\left(\begin{array}{cc}
\vec{\sigma} & 0 \\
0 & \vec{\sigma}
\end{array}\right)
$$

From the above operator we can see that, although it is a $4 \times 4$ matrix differential one, it is diagonal in block of $2 \times 2$ matrices, which means that the two upper components of Dirac spinor interact with the gravitational field in the similar way as the two lower components. 
The complete differential operator in Eq. (11) is

$$
\mathcal{L}=-\frac{1}{\alpha^{2}} \partial_{t}^{2}+\frac{\alpha^{2}}{r^{2}} \partial_{r} r^{2} \partial_{r}-\frac{1}{r^{2}} \vec{L}^{2}-\frac{1-\alpha}{r^{2}}(1+\vec{\Sigma} \cdot \vec{L})-M^{2}
$$

Now we shall consider the case where the fermionic field has no mass. In this case we are able to obtain a closed expression for the fermionic propagator as follows.

The system which we shall consider consists of a massless left-handed fermionic field in a global monopole manifold. The Dirac equation reduces to a $2 \times 2$ matrix differential one, as shown below:

$$
i \not D L \chi=0
$$

where

$$
\not D_{L}=i\left[\frac{1}{\alpha} \partial_{t}-\alpha \sigma^{(r)} \partial_{r}-\frac{1}{r} \sigma^{(\theta)} \partial_{\theta}-\frac{1}{r \sin \theta} \sigma^{(\varphi)} \partial_{\varphi}+\frac{1-\alpha}{r} \sigma^{(r)}\right]
$$

with $\sigma^{(r)}=\vec{\sigma} \cdot \hat{\vec{r}}, \sigma^{(\theta)}=\vec{\sigma} \cdot \hat{\vec{\theta}}, \sigma^{(\varphi)}=\vec{\sigma} \cdot \hat{\vec{\varphi}}$, where $\hat{\vec{r}}, \hat{\vec{\theta}}$ and $\hat{\vec{\varphi}}$ are the standard unit vectors along the three spatial directions in spherical coordinates.

The Feynman two-component propagator obeys the equation

$$
i \not D_{L} \mathcal{S}_{\mathcal{F}}\left(x, x^{\prime}\right)=\frac{1}{\sqrt{-g}} \delta^{(4)}\left(x-x^{\prime}\right) I_{2}
$$

and can be given in terms of the bispinor $\mathcal{G}_{\mathcal{F}}$ by

$$
\mathcal{S}_{\mathcal{F}}\left(x, x^{\prime}\right)=i \not D \mathcal{G}_{\mathcal{F}}\left(x, x^{\prime}\right)
$$

where now, $\mathcal{G}_{\mathcal{F}}\left(x, x^{\prime}\right)$ obeys the $2 \times 2$ differential equation

$$
\overline{\mathcal{L}} \mathcal{G}_{\mathcal{F}}\left(x, x^{\prime}\right)=-\frac{1}{\sqrt{-g}} \delta^{(4)}\left(x-x^{\prime}\right) I_{2}
$$

with

$$
\overline{\mathcal{L}}=-\frac{1}{\alpha^{2}} \partial_{t}^{2}+\frac{\alpha^{2}}{r^{2}} \partial_{r} r^{2} \partial_{r}-\frac{1}{r^{2}} \vec{L}^{2}-\frac{1-\alpha}{r^{2}}(1+\vec{\sigma} \cdot \vec{L})
$$

The vacuum average value of the energy-momentum tensor may be expressed in terms of the Euclidean Green function which is simpler than the ordinary Feynman Green function. They are connected by the relation 11$] \mathcal{G}_{\mathcal{E}}\left(\tau, \vec{r} ; \tau^{\prime}, \overrightarrow{r^{\prime}}\right)=$ $-i \mathcal{G}_{\mathcal{F}}\left(x, x^{\prime}\right)$, where $t=i \tau$. In the following we shall consider the Euclidean Green function. In order to find a solution for the bispinor $\mathcal{G}_{\mathcal{E}}\left(x, x^{\prime}\right)$, we shall obtain the solution for the eigenvalue equation

$$
\overline{\mathcal{L}} \phi_{\lambda}(x)=-\lambda^{2} \phi_{\lambda}(x)
$$

with $\lambda^{2} \geq 0$, so we can write

$$
\mathcal{G}_{\mathcal{E}}\left(x, x^{\prime}\right)=\sum_{\lambda} \frac{\phi_{\lambda}(x) \phi_{\lambda}^{+}\left(x^{\prime}\right)}{\lambda^{2}}
$$

Due to fact that our operator (25) is self-adjoint, the set of its eigenfunctions constitutes a basis for the Hilbert space associated with two-component spinors. Moreover, because operator $\overline{\mathcal{L}}$ is a parity even operator, its eigenfunctions present a defined parity, so the normalized eigenfunctions can be written as:

$$
\begin{aligned}
& \phi_{\lambda}^{(1)}(x)=e^{-i E \tau} f^{(1)}(r) \varphi_{j, m_{j}}^{(1)}(\theta, \varphi), \\
& \phi_{\lambda}^{(2)}(x)=e^{-i E \tau} f^{(2)}(r) \varphi_{j, m_{j}}^{(2)}(\theta, \varphi)
\end{aligned}
$$

where $\varphi_{j, m_{j}}^{(k)}$, with $k=1,2$, are the spinor spherical harmonics which are eigenfunctions of the operators $\vec{L}^{2}$ and $\vec{\sigma} \cdot \vec{L}$ as shown below: 


$$
\begin{aligned}
\vec{L}^{2} \varphi_{j, m_{j}}^{(1,2)} & =l(l+1) \varphi_{j, m_{j}}^{(1,2)}, \\
\vec{\sigma} \vec{L} \varphi_{j, m_{j}}^{(1,2)} & =-\left(1+\kappa^{(1,2)}\right) \varphi_{j, m_{j}}^{(1,2)},
\end{aligned}
$$

with $\kappa^{(1)}=-(l+1)=-(j+1 / 2)$ and $\kappa^{(2)}=l=j+1 / 2$. Explicit form of above standard function are given in Ref. [12], for example.

Substituting $\phi_{\lambda}^{(1,2)}$ into 26 , we obtain the following eigenfunctions

$$
\begin{aligned}
\phi_{\lambda}^{(k)}(x) & =\sqrt{\frac{p}{2 \pi r}} e^{-i E \tau} J_{\nu_{k}}(p r) \varphi_{j, m_{j}}^{(k)}, \\
\lambda^{2} & =E^{2} / \alpha^{2}+\alpha^{2} p^{2}, \\
\nu_{1} & =\frac{l+1}{\alpha}-\frac{1}{2}, \nu_{2}=\frac{l}{\alpha}+\frac{1}{2},
\end{aligned}
$$

where $J_{\nu}$ is the Bessel function of first kind. We can see that for Minkowski spacetime where $\alpha=1$ we have $\nu_{1}=\nu_{2}=l+1 / 2$.

Now we are in condition to obtain the bispinor $\mathcal{G}_{\mathcal{E}}$ which is given by:

$$
\mathcal{G}_{\mathcal{E}}\left(x, x^{\prime}\right)=\int_{-\infty}^{+\infty} d E \int_{0}^{\infty} d p \sum_{j, m_{j}} \frac{\phi_{\lambda}^{(1)}(x) \phi_{\lambda}^{(1)+}\left(x^{\prime}\right)+\phi_{\lambda}^{(2)}(x) \phi_{\lambda}^{(2)+}\left(x^{\prime}\right)}{E^{2} / \alpha^{2}+\alpha^{2} p^{2}} .
$$

Finally, substituting our result for the two-component spinor $\phi_{\lambda}^{(k)}$ given by (31) and (32) into (33) we obtain, with the help of Ref. [13], an expression for the Euclidean Green function

$$
\mathcal{G}_{\mathcal{E}}\left(x, x^{\prime}\right)=\frac{1}{2 \pi r r^{\prime}} \sum_{j, m_{j}}\left[Q_{\nu_{1}-1 / 2}(u) C_{j, m_{j}}^{(1)}\left(\Omega, \Omega^{\prime}\right)+Q_{\nu_{2}-1 / 2}(u) C_{j, m_{j}}^{(2)}\left(\Omega, \Omega^{\prime}\right)\right],
$$

where $Q_{\nu}(u)$ are the Legendre functions of second kind; $u=1+\left(\alpha^{4} \triangle \tau^{2}+\triangle r^{2}\right) / 2 r r^{\prime}$ and $C_{j, m_{j}}^{(k)}\left(\Omega, \Omega^{\prime}\right)=$ $\varphi_{j, m_{j}}^{(k)}(\Omega) \varphi_{j, m_{j}}^{(k)+}\left(\Omega^{\prime}\right)$. Again we can see that in Minkowski limit, for $\alpha=1, \nu_{1}=\nu_{2}=l+1 / 2$ and we get

$$
\mathcal{G}_{\mathcal{E}}\left(x, x^{\prime}\right)=\frac{1}{8 \pi^{2}} \frac{1}{\sigma_{M}\left(x, x^{\prime}\right)} I_{2},
$$

where we have used the relation envolving the sum of Legendre functions and polynomials 13 . Here $\sigma_{M}\left(x, x^{\prime}\right)=$ $\left(\triangle \tau^{2}+\left(\vec{r}-\vec{r}^{\prime}\right)^{2}\right) / 2$ is one-half the square of geodesic distance between $x$ and $x^{\prime}$ in the flat Euclidean space.

Now in order to obtain the Green function $\mathcal{S}_{\mathcal{F}}$ let us go back to Eq. (34). Using (23) we have

$$
\mathcal{S}_{\mathcal{F}}\left(x, x^{\prime}\right)=i\left[\frac{1}{\alpha} \partial_{t}-\alpha \sigma^{(r)} \partial_{r}+\frac{1}{r} \sigma^{(r)} \vec{\sigma} \cdot \vec{L}+\frac{1-\alpha}{r} \sigma^{(r)}\right] \mathcal{G}_{\mathcal{F}}\left(x, x^{\prime}\right) .
$$

\section{VACUUM EXPECTATION VALUES}

Now let us proceed with the calculation of the vacuum expectation value (VEV) of the energy-momentum tensor. Initially, we would like to discuss the general structure of this tensor. As it will be seen later and has already been discussed in scalar case in Ref. [7], the renormalized VEV of the energy-momentum tensor has the following structure

$$
<T_{\mu}^{\nu}>^{r e n}=\frac{1}{8 \pi^{2} r^{4}}\left[A_{\mu}^{\nu}+B_{\mu}^{\nu} \ln \frac{\mu r}{\alpha}\right],
$$

where the tensors $A_{\mu}^{\nu}$ and $B_{\mu}^{\nu}$ depend on the metric parameter $\alpha$, only. The scaling parameter $\mu$ appears after renormalization procedure. Obviously these tensors are diagonal and the component $A_{\theta}^{\theta}=A_{\varphi}^{\varphi}$ and $B_{\theta}^{\theta}=B_{\varphi}^{\varphi}$ due to spherical symmetry of the problem. Therefore we have six unknown components. The renormalized VEV of the energy-momentum tensor must be conserved, i.e.,

$$
<T_{\mu}^{\nu}>_{; \nu}^{r e n}=0
$$


and gives the right conformal anomaly [14, which for massles spinor two-component field, reads [15]

$$
<T_{\mu}^{\mu}>^{r e n}=\frac{1}{16 \pi^{2}} \operatorname{tr} a_{2}=\frac{T}{8 \pi^{2} r^{4}} .
$$

Taking into account Eqs. (38), (39) we may express tensors $A_{\mu}^{\nu}$ and $B_{\mu}^{\nu}$ in terms of the zero-zero components $A_{0}^{0}, B_{0}^{0}$ and the trace $T$ by:

$$
\begin{aligned}
& A_{\mu}^{\nu}=\operatorname{diag}\left(A_{0}^{0} ;-T+A_{0}^{0}+B_{0}^{0} ; T-A_{0}^{0}-\frac{1}{2} B_{0}^{0} ; T-A_{0}^{0}-\frac{1}{2} B_{0}^{0}\right), \\
& B_{\mu}^{\nu}=B_{0}^{0} \operatorname{diag}(1 ; 1 ;-1 ;-1) .
\end{aligned}
$$

Therefore our problem now is to obtain the zero-zero components $A_{0}^{0}$ and $B_{0}^{0}$. Using the point-splitting approach, the VEV of the energy-momentum tensor for spinor field has the following form (see Ref. [11] for example):

$$
<T_{\mu \nu}>=\frac{1}{4} \lim _{x^{\prime} \rightarrow x} \operatorname{tr}\left[\sigma_{\mu}\left(\nabla_{\nu}-\nabla_{\nu^{\prime}}\right)+\sigma_{\nu}\left(\nabla_{\mu}-\nabla_{\mu^{\prime}}\right)\right] \mathcal{S}_{\mathcal{F}}\left(x, x^{\prime}\right),
$$

by means of which we have

$$
<T_{0}^{0}>=\frac{i}{\alpha^{2}} \lim _{x^{\prime} \rightarrow x} \partial_{t}^{2} \operatorname{tr}\left(\mathcal{G}_{\mathcal{F}}\left(x, x^{\prime}\right)\right)=-\frac{1}{\alpha^{2}} \lim _{x^{\prime} \rightarrow x} \partial_{\tau}^{2} \operatorname{tr}\left(\mathcal{G}_{\mathcal{E}}\left(x, x^{\prime}\right)\right) .
$$

The first term with time derivative in Eq. (36) gives non-zero contribution in the zero-zero component of the energymomentum tensor, only. Indeed, the Euclidean Green function (34) is proportional to the unit matrix $I_{2}$ after taking the coincidence limit $\Omega^{\prime}=\Omega$ and sum over $m_{j}$. The same is true for the third term in (36) due to Eq. (30). We obtain zero contribution from these terms because Pauli matrices are traceless. In above expression we have used also that the Green function (34) depends on the $\triangle \tau$ and hence $\partial_{\tau^{\prime}} \mathcal{G}_{\mathcal{E}}=-\partial_{\tau} \mathcal{G}_{\mathcal{E}}$.

Taking the coincidence limit $\Omega^{\prime}=\Omega, r^{\prime}=r$ into Eq. (34), summing over $m_{j}$ and after using the integral representation for the Legendre function [13], it is possible to develop the sum over $j$, which is geometric series and we arrive at the following formula for the Euclidean Green function

$$
8 \pi^{2} \mathcal{G}_{\mathcal{E}}(\triangle \tau, r)=\frac{1}{2 r^{2}} \int_{b}^{\infty} \frac{d x}{\sqrt{x^{2}-b^{2}}} \frac{1}{\operatorname{sh}^{2}\left(\frac{\operatorname{arsh} x}{\alpha}\right)} I_{2}
$$

where the function $b$ may be expressed in terms of the one-half of the square geodesic distance $\sigma$ in $\tau$ direction

$$
b^{2}=\frac{\alpha^{2}}{2 r^{2}} \sigma=\frac{\alpha^{2}}{2 r^{2}}\left(\frac{\alpha^{2} \triangle \tau^{2}}{2}\right) .
$$

In the case when only the angular variable $\Omega^{\prime}=\Omega$ coincides, we shall get the same formula for Green function (44) with $\sigma=\left(\alpha^{2} \triangle \tau^{2}+\triangle r^{2} / \alpha^{2}\right) / 2$ and $r^{2} \rightarrow r r^{\prime}$.

The Green function (44) is divergent in the coincidence limit $b \rightarrow 0$. For renormalization in the massless case it is more suitable to subtract from the Green function (44) the Green function in the Hadamard form given below (see Refs. [16. 9])

$$
8 \pi^{2} \mathcal{G}_{\mathcal{E}}^{\mathcal{H}}\left(x, x^{\prime}\right)=\triangle^{1 / 2}\left\{\frac{a_{0}}{\sigma}-\frac{3 a_{2}}{8} \sigma+\left[-\frac{a_{1}}{2}+\frac{a_{2}}{4} \sigma\right] \ln \left(\frac{1}{8} \mu^{2} \sigma\right)\right\} .
$$

In order to obey the conservation law (38) we must subtract from the energy-momentum tensor additional contribution $g_{\mu \nu} \operatorname{tr} a_{2} / 64 \pi^{2}$ according to Wald [9]. The general form of the coefficients $a_{k}$ may be found in Ref. [17] and for the global monopole spacetime they have the following form

$$
\triangle=1, a_{0}=I_{2}, a_{1}=-\frac{1-\alpha^{2}}{6 r^{2}} I_{2}, a_{2}=-\frac{1-\alpha^{4}}{60 r^{4}} I_{2} .
$$

From our expression for the Green function (44), it is possible to extract all divergencies in manifest form. To do this let us consider the rhs of Eq. (44). We divide the integral in two parts - first one from $b$ to unit and second from unit to infinity. In the first part we have the form below

$$
V_{1}=\frac{1}{2 r^{2}} \int_{b}^{1} \frac{d x}{\sqrt{x^{2}-b^{2}}} \frac{1}{\operatorname{sh}^{2}\left(\frac{\operatorname{arsh} x}{\alpha}\right)} I_{2} .
$$


Subtracting and adding into the integrand the three first terms of the power series of the function

$$
\frac{1}{\operatorname{sh}^{2}\left(\frac{\operatorname{arsh} x}{\alpha}\right)}=\frac{\alpha^{2}}{x^{2}}-\frac{1-\alpha^{2}}{3}+\frac{1-\alpha^{4}}{15} \frac{x^{2}}{\alpha^{2}}+\ldots
$$

we get

$$
V_{1}=V_{1}^{f i n}+V_{1}^{\text {div }}
$$

The first term is given by expression

$$
V_{1}^{f i n}=\frac{1}{2 r^{2}} \int_{b}^{1} \frac{d x}{\sqrt{x^{2}-b^{2}}}\left\{\frac{1}{\operatorname{sh}^{2}\left(\frac{\operatorname{arsh} x}{\alpha}\right)}-\frac{\alpha^{2}}{x^{2}}+\frac{1-\alpha^{2}}{3}-\frac{1-\alpha^{4}}{15} \frac{x^{2}}{\alpha^{2}}\right\} I_{2},
$$

and its two derivatives with respect to time $\tau$ are finite in the coincidence limit $b \rightarrow 0$. All divergencies are contained in the second part of Eq. (50) which has the form

$$
V_{1}^{d i v}=\frac{1}{2 r^{2}}\left[\frac{\alpha^{2}}{b^{2}} \sqrt{1-b^{2}}-\frac{1-\alpha^{2}}{3} \ln \frac{1+\sqrt{1-b^{2}}}{b}+\frac{1-\alpha^{4}}{30 \alpha^{2}}\left\{\sqrt{1-b^{2}}+b^{2} \ln \frac{1+\sqrt{1-b^{2}}}{b}\right\}\right] I_{2} .
$$

Expanding this expression in the power series over $b$ up to the terms which will survive after taking the second derivative and coincidence limit, we may represent it in the form

$$
V_{1}^{d i v}=\left\{\frac{1}{\sigma}+\frac{1}{2 r^{2}}\left[-\frac{\alpha^{2}}{2}+\frac{1-\alpha^{4}}{30 \alpha^{2}}\right]+\left[-\frac{\alpha^{4}}{32 r^{4}}+\frac{\alpha^{2}\left(1-\alpha^{2}\right)}{48 r^{4}}+\frac{a_{2}}{4}\right] \sigma+\left[-\frac{a_{1}}{2}+\frac{a_{2}}{4} \sigma\right] \ln \frac{\alpha^{2} \sigma}{8 r^{2}}\right\} I_{2} .
$$

We see that the divergent part of this expression has the Hadamard form given by Eq.46. The next powers in the expansion (49) will give finite contribution to the energy-momentum tensor.

The second part

$$
V_{2}=\frac{1}{2 r^{2}} \int_{1}^{\infty} \frac{d x}{\sqrt{x^{2}-b^{2}}} \frac{1}{\operatorname{sh}^{2}\left(\frac{\operatorname{arsh} x}{\alpha}\right)} I_{2}
$$

does not contain divergencies and it may be easily expanded in terms of $b$

$$
V_{2}=\frac{1}{2 r^{2}} \int_{1}^{\infty} \frac{d x}{x} \frac{1}{\operatorname{sh}^{2}\left(\frac{\operatorname{arsh} x}{\alpha}\right)} I_{2}+\frac{\alpha^{2} \sigma}{8 r^{4}} \int_{1}^{\infty} \frac{d x}{x^{3}} \frac{1}{\operatorname{sh}^{2}\left(\frac{\operatorname{arsh} x}{\alpha}\right)} I_{2}+O\left(\sigma^{2}\right) .
$$

Taking into account all above formulas we obtain the following expression for the renormalized Green function

$$
\begin{aligned}
8 \pi^{2} \mathcal{G}_{\mathcal{E}}{ }^{r e n} & =8 \pi^{2}\left[\mathcal{G}_{\mathcal{E}}(\triangle \tau, r)-\mathcal{G}_{\mathcal{E}}^{\mathcal{H}}(\triangle \tau, r)\right] \\
& =V_{1}^{f i n}+V_{2}+\left\{\frac{1}{2 r^{2}}\left[-\frac{\alpha^{2}}{2}+\frac{1-\alpha^{4}}{30 \alpha^{2}}\right]+\left[-\frac{\alpha^{4}}{32 r^{4}}+\frac{\alpha^{2}\left(1-\alpha^{2}\right)}{48 r^{4}}+\frac{5 a_{2}}{8}\right] \sigma+\left[a_{1}-\frac{a_{2}}{2} \sigma\right] \ln \frac{\mu r}{\alpha}\right\} I_{2},
\end{aligned}
$$

where $V_{1}^{f i n}$ and $V_{2}$ are given by eqs. (51) and (54), respectively. Plugging this expression for the renormalized Green function into Eq. (43) we obtain the following formulas for the zero-zero components of $A_{0}^{0}$ and $B_{0}^{0}$ (see Appendix (A)

$$
\begin{aligned}
& A_{0}^{0}=-\frac{1}{8}\left\{\int_{0}^{1} \frac{d x}{x}\left[\left(\frac{\alpha^{2}}{\operatorname{sh}^{2}\left(\frac{\operatorname{arsh} x}{\alpha}\right)}\right)^{\prime \prime}-\frac{6 \alpha^{4}}{x^{4}}-\frac{2\left(1-\alpha^{4}\right)}{15}\right]+\int_{1}^{\infty} \frac{d x}{x}\left[\left(\frac{\alpha^{2}}{\operatorname{sh}^{2}\left(\frac{\operatorname{arsh} x}{\alpha}\right)}\right)^{\prime \prime}-\frac{6 \alpha^{4}}{x^{4}}\right]\right\}, \\
& B_{0}^{0}=\frac{r^{4} \operatorname{tr} a_{2}}{2}=-\frac{1-\alpha^{4}}{60},
\end{aligned}
$$

where the prime means the derivative with respect to $x$. In this expression we have already taken into account additional contribution $-\operatorname{tr} a_{2} / 64 \pi^{2}$.

Now we have explicit expressions for zero-zero components $A_{0}^{0}$ and $B_{0}^{0}$ for arbitrary values of $\alpha$. Let us analyse $A_{0}^{0}$ component (see Appendix B for details). 
1. For large solid angle deficit $(\alpha \ll 1)$ :

$$
A_{0}^{0} \sim-\frac{1}{60} \ln \alpha+C_{0}, C_{0}=0.0104 .
$$

2. For small solid angle deficit (excess) $(|\alpha-1| \ll 1)$ :

$$
A_{0}^{0} \sim C_{1}(1-\alpha), C_{1}=0.0773 .
$$

3. The large solid angle excess $(\alpha \gg 1)$ :

$$
A_{0}^{0} \sim-C_{\infty} \alpha^{4}, C_{\infty}=0.0173 .
$$

The numerical calculation of $A_{0}^{0}$ is shown in Fig.1]
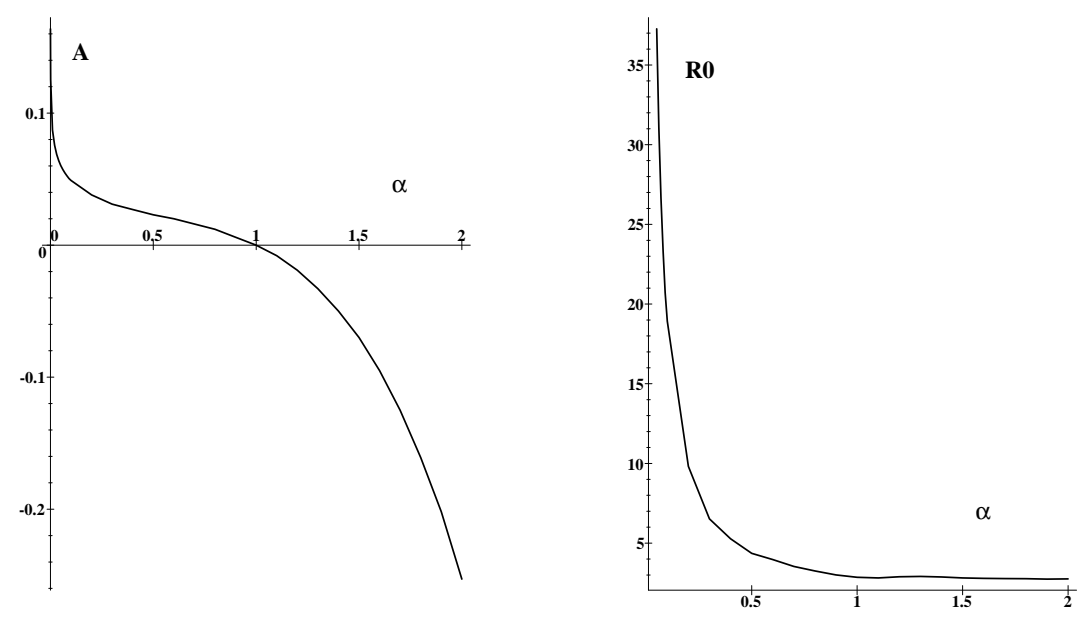

FIG. 1. Zero-zero component $A_{0}^{0}$ and distance $R_{0}$ where the component $\left\langle T_{0}^{0}\right\rangle^{r e n}$ of energy-momentum tensor changes its sign, are plotted as function of the metric parameter $\alpha$

Now, we would like to compare our results with the similar one for the VEV of the energy-momentum tensor of the massless spinor field in the infinitely thin cosmic string spacetime with line element, in cylindrical coordinate system, given by

$$
d s^{2}=-d t^{2}+d \rho^{2}+\frac{\rho^{2}}{\nu^{2}} d \varphi^{2}+d z^{2} .
$$

In Ref. 18] has been obtained the following result

$$
T_{\mu}^{\nu}=\frac{\left(\nu^{2}-1\right)\left(7 \nu^{2}+17\right)}{1440 \cdot 4 \pi^{2} \rho^{4}} \operatorname{diag}(1 ; 1 ; 1 ;-3) .
$$

In this case there is no the logarithmic contribution because this spacetime is locally flat. Therefore we may compare now the $A_{0}^{0}$ given by Eq. (57) for global monopole spacetime with the analogous one for infinitely thin cosmic string spacetime which is given by expression

$$
A_{0(c s)}^{0}=\frac{\left(\nu^{2}-1\right)\left(7 \nu^{2}+17\right)}{720}
$$

To do so, we have to change the radial variable $r=\alpha \rho$ because in this coordinate system the section $\theta=\pi / 2$ of the monopole spacetime (何) coincides with section $z=$ const of the infinitely thin cosmic string spacetime (62) and the 
parameter $\alpha=1 / \nu$. Therefore we may compare $A_{0(c s)}^{0}(\nu)$ with $\left(A_{0(g m)}^{0}(\alpha) / \alpha^{4}\right)_{\alpha=1 / \nu}$. We have qualitative agreement of both these quantities. For $\nu \ll 1$ they go to negative constant; for small angle deficit they are proportional to $\nu-1$; for $\nu \gg 1$ we have $A_{0(c s)}^{0} \sim \nu^{4}$ and $\left[\nu^{4} A_{0(g m)}^{0}(1 / \nu)\right] \sim \nu^{4}\left[\ln \nu+60 C_{0}\right]$. Both of these quantities change the sign at point $\nu=1$.

The dependence of $\left\langle T_{0}^{0}>^{\text {ren }}\right.$ on the distance from origin in the global monopole spacetime is competely different from the case of the infinitely thin cosmic string due to the logarithmic term. Let us consider the physical distance $R=\mu r / \alpha$ measured in a mass scale $\mu$. Then, the $<T_{0}^{0}>^{r e n}$ measured in units $\mu^{4}$ has the form

$$
8 \pi^{2} \mu^{-4}<T_{0}^{0}>^{r e n}=\frac{\alpha^{4}-1}{60 \alpha^{4} R^{4}} \ln \frac{R}{R_{0}}
$$

where $R_{0}=\exp \left(-A_{0}^{0} / B_{0}^{0}\right)$. In the case $\alpha<1$ the energy density $<T_{0}^{0}>^{r e n}$ is positive in the domain $R \in\left(0, R_{0}\right)$; it changes the sign in the point $R=R_{0}$ and goes, through the minimum at point $R_{*}=R_{0} e^{1 / 4}$, to zero in infinity. In the case $\alpha>1$ we have opposite picture with maximum at the point $R_{*}$. The dependence of $R_{0}$ on the $\alpha$ is shown in the Fig.1. For small $\alpha$ it is proportional to $1 / \alpha$ and it goes to constant for great $\alpha$.

\section{CONCLUSION}

In this paper we have considered the quantum, a 1/2-spin left-handed field in the background of a pointlike global monopole described by the metric tensor given in (位). More specifically, we have obtained the complete Feynman propagator, expressed in terms of a bispinor, in a closed form. Differently from the results obtained in Ref. [7 for scalar field, in this analysis there appears two effective angular total quantum numbers, which we call by $\nu_{1}$ and $\nu_{2}$. These angular total quantum numbers are related with the explicit properties of the spinor harmonics, $\varphi_{j, m_{j}}^{(1)}$ and $\varphi_{j, m_{j}}^{(2)}$ and they have the following form $\nu_{1}=(l+1) / \alpha-1 / 2$ and $\nu_{2}=l / \alpha+1 / 2$. For the scalar case the effective orbital quantum numbers take a simple form only under specific situation [7], namely for $\xi=1 / 8$.

The main goal of this work was to obtain the renormalized vacuum expectation value of the energy-momentum tensor. Because all components of the VEV of the energy-momentum tensor can be expressed in terms of its zero-zero component, we used only the Euclidean Green function at the coincidence limit $\Omega^{\prime}=\Omega$ and $r^{\prime}=r$. In this case, in order to obtain the regularized expression for $\left\langle T_{0}^{0}\right\rangle$, we were able to subtract all divergencies in a manifest form.

After the renormalization, the Green function (56) depends on the scale parameter $\mu$ which leads to logarithm contribution to the energy-momentum tensor given by Eq.(37). But, as it was noted in Ref. [7] the one-loop Einstein equations

$$
R_{\mu \nu}-\frac{1}{2} R g_{\mu \nu}+\epsilon_{1}^{(1)} H_{\mu \nu}+\epsilon_{2}^{(2)} H_{\mu \nu}=8 \pi\left\{T_{\mu \nu}^{c l a s}+<T_{\mu \nu}>^{r e n}\right\}
$$

do not depend on this parameter due to the renormalization group equations for the coefficients $\epsilon_{1}$ and $\epsilon_{2}$. Any variation of the scale parameter $\mu$ may be absorbed by variation of the $\epsilon_{k}$.

Taking into account the conservation law (38), the expression for conformal anomaly (39) and the spherical symmetry of problem we expressed all components of energy-momentum tensor in terms of zero-zero component (41) which has the form

$$
<T_{0}^{0}>^{r e n}=\frac{1}{8 \pi^{2} r^{4}}\left[A_{0}^{0}+B_{0}^{0} \ln \frac{\mu r}{\alpha}\right],
$$

where $A_{0}^{0}$ and $B_{0}^{0}$ are given by eqs. (57) and (58). The component $A_{0}^{0}$ depicted in Fig.1 as a function of $\alpha$, and it is qualitatively agreed with similar one in the infinitely thin cosmic string spacetime.

The scaling parameter $\mu$ leads to the logarithmic contribution in the energy-momentum tensor (67). For this reason $<T_{0}^{0}>^{r e n}$ changes its sign in some point $R_{0}$ and has the extremum at the point $R_{*}=R_{0} e^{1 / 4}$. The dependence of $R_{0}$ on the $\alpha$ is depicted in the Fig. 11.

Before we finish this paper we would like to make a brief comment about some results previously obtained in the literature related with quantum calculation of the energy-momentum tensor $T_{\mu}^{\nu}$ in the spacetime of a pointlike global monopole. Hiscock, in Ref. [8], using general consideration, obtained a formal expression for the vacuum expectation value of the energy-momentum tensor for an arbitrary collection of conformal massless fields in this manifold. Later, Mazzitelly and Lousto, in Ref. [7], developed the explicit calculation for renormalized vacuum average of the square of a massless scalar quantum field and also, by general consideration, infered the structure for $T_{\mu}^{\nu}$ which disagrees with the Hiscock's results and manifestly depends on the scale parameter $\mu$. This is in agreement with general 
consideration by Wald in Ref. 9. In our paper we obtained the explicit expression for vacuum average value of the energy-momentum tensor for a massless fermionic field in this manifold, giving the complete information about this tensor in terms of $A_{0}^{0}$ and $B_{0}^{0}$. We also have analyzed the behaviour of these functions with the parameter $\alpha$, and the dependence of $T_{0}^{0}$ with the physical distance $R$. Finally we want to say that our expression for VEV of the energy-momentum tensor depend on the scale parameter $\mu$, in agreement with Ref. [9], due to the appearance of a logarithm term, which is a consequence of the regularization procedure. This term is responsible for the changing in the sign of the vacuum energy-momentum tensor when we vary the physical distance $R$.

The explicit expression for the massless left-handed two-component spinor Green function, is obtained in the background of global monopole spacetime. It is our interest to develop a similar calculation for the massive case and also obtain the vacuum average for the energy-momentum tensor.

\section{ACKNOWLEDGMENTS}

NK is grateful to Departamento de Física, Universidade Federal da Paraiba (Brazil) where this work was done, for hospitality. His work was supported in part by CAPES and in part by the Russian Fund for Basic Research, grant No 97-02-16318.

ERBM and VBB also would like to thank the Conselho Nacional de Desenvolvimento Cientifico e Tecnológico $(\mathrm{CNPq})$.

\section{APPENDIX A:}

In order to find derivatives of $V_{1}^{\text {fin }}$ with respect to $b$ we have to change variable $x \rightarrow b x$ because integrand in $V_{1}^{f i n}$ is divergent at the point $x=b$. The second derivative has the form below

$$
2 r^{2} \frac{d^{2} V_{1}^{\text {fin }}}{d b^{2}}=\frac{1-b^{2}}{b^{2}\left(1-b^{2}\right)^{3 / 2}} f(1)-\frac{1}{b^{2} \sqrt{1-b^{2}}} f^{\prime}(1)+\frac{1}{b^{2}} \int_{b}^{1} \frac{d x x^{2}}{\sqrt{x^{2}-b^{2}}} f^{\prime \prime}(x),
$$

where

$$
f(x)=\frac{1}{\operatorname{sh}^{2}\left(\frac{\operatorname{arsh} x}{\alpha}\right)}-\frac{\alpha^{2}}{x^{2}}+\frac{1-\alpha^{2}}{3}-\frac{1-\alpha^{4}}{15} \frac{x^{2}}{\alpha^{2}} .
$$

The last integral may be represented in the following form

$$
\frac{1}{b^{2}} \int_{b}^{1} \frac{d x x^{2}}{\sqrt{x^{2}-b^{2}}} f^{\prime \prime}(x)=\frac{\sqrt{1-b^{2}}}{b^{2}}\left(f^{\prime}(1)-f(1)\right)+\int_{b}^{1} \frac{d x}{\sqrt{x^{2}-b^{2}}}\left[f^{\prime \prime}(x)-\frac{1}{x} f^{\prime}(x)+\frac{1}{x^{2}} f(x)\right] .
$$

Taking into account the above expression and putting $b=0$ we have that

$$
2 r^{2}{\frac{d^{2} V_{1}^{f i n}}{d b^{2}}}_{b=0}=-f^{\prime}(1)+\int_{0}^{1} \frac{d x}{x}\left[f^{\prime \prime}(x)-\frac{1}{x} f^{\prime}(x)+\frac{1}{x^{2}} f(x)\right] .
$$

Integrating by parts the term with second derivative we arrive at the expression

$$
{\frac{d^{2} V_{1}^{f i n}}{d b^{2}}}_{b=0}=\frac{1}{2 r^{2}} \int_{0}^{1} \frac{d x}{x^{3}} f(x) .
$$

The second derivative of $V_{2}$ may be easily found from Eq. (54) and is given by

$$
{\frac{d^{2} V_{2}}{d b^{2}}}_{b=0}=\frac{1}{2 r^{2}} \int_{1}^{\infty} \frac{d x}{x^{3}} \frac{1}{\operatorname{sh}^{2}\left(\frac{\operatorname{arsh} x}{\alpha}\right)} .
$$

Using the above formulas in $(56)$ and $(43)$, we get the following expression for $<T_{0}^{0}>^{r e n}$

$$
\begin{aligned}
8 \pi^{2}<T_{0}^{0}>^{r e n}= & -\frac{\alpha^{2}}{4 r^{4}}\left\{\int_{0}^{1} \frac{d x}{x^{3}}\left[\frac{1}{\operatorname{sh}^{2}\left(\frac{\operatorname{arsh} x}{\alpha}\right)}-\frac{\alpha^{2}}{x^{2}}+\frac{1-\alpha^{2}}{3}-\frac{1-\alpha^{4}}{15} \frac{x^{2}}{\alpha^{2}}\right]\right. \\
& \left.+\int_{1}^{\infty} \frac{d x}{x^{3}}\left[\frac{1}{\operatorname{sh}^{2}\left(\frac{\operatorname{arsh} x}{\alpha}\right)}-\frac{\alpha^{2}}{x^{2}}+\frac{1-\alpha^{2}}{3}\right]\right\}+\frac{\operatorname{tr} a_{2}}{2} \ln \frac{\mu r}{\alpha}-\frac{5}{8} \operatorname{tr} a_{2}-\frac{1}{8} \operatorname{tr} a_{2} .
\end{aligned}
$$


Because we have used for renormalization of the Green function in the Hadamard form (46) the last term has been added in order to obey conservation law (38) according to Wald [9] . Integrating two times by parts we arrive at the formula (57); the last two terms in $(\mathrm{A} 8 \mathrm{~B})$ are cancelled.

\section{APPENDIX B:}

Here we will analyse $A_{0}^{0}$ component in three domains : $\alpha \ll 1,|\alpha-1| \ll 1$ and $\alpha \gg 1$. $A_{0}^{0}$ has the form below

$$
A_{0}^{0}=-\frac{1}{8}\left\{\int_{0}^{1} \frac{d x}{x} f_{2}+\int_{1}^{\infty} \frac{d x}{x} f_{1}\right\}
$$

where

$$
\begin{aligned}
& f_{1}=\frac{2 \alpha x}{\left(1+x^{2}\right)^{3 / 2}} \frac{\operatorname{ch} y}{\operatorname{sh}^{3} y}-\frac{2}{1+x^{2}}\left(\frac{1}{\operatorname{sh}^{2} y}-\frac{3 \operatorname{ch}^{2} y}{\operatorname{sh}^{4} y}\right)-6 \frac{\alpha^{4}}{x^{4}}, \\
& f_{2}=f_{1}-\frac{2}{15}\left(1-\alpha^{4}\right), y=\frac{1}{\alpha} \operatorname{arsh} x .
\end{aligned}
$$

1. Case $\alpha \ll 1$.

Here we represent $A_{0}^{0}$ in the following form

$$
A_{0}^{0}=-\frac{1}{8}\left\{\int_{0}^{\alpha} \frac{d x}{x} f_{3}+\int_{\alpha}^{\infty} \frac{d x}{x} f_{2}-\frac{2}{15}\left(1-\alpha^{4}\right) \int_{\alpha}^{1} \frac{d x}{x}\right\} .
$$

The first and second integrals are finite in the limit $\alpha \rightarrow 0$ (after changing $x \rightarrow \alpha x$ ) and the last integral gives logarithm term. Putting together we obtain

$$
\begin{aligned}
& A_{0}^{0}=-\frac{1}{60} \ln \alpha+C_{0} \\
& C_{0}=\frac{1}{4}\left\{\int_{0}^{1} \frac{d x}{x}\left(\frac{1}{\operatorname{sh}^{2} x}-\frac{3 \operatorname{ch}^{2} x}{\operatorname{sh}^{4} x}+\frac{3}{x^{4}}+\frac{1}{15}\right)+\int_{1}^{\infty} \frac{d x}{x}\left(\frac{1}{\operatorname{sh}^{2} x}-\frac{3 \operatorname{ch}^{2} x}{\operatorname{sh}^{4} x}+\frac{3}{x^{4}}\right)\right\}=0.0104 .
\end{aligned}
$$

2. Case $|\alpha-1| \ll 1$. There is no problem to expand $A_{0}^{0}$ near the point $\alpha=1$ and we get:

$$
A_{0}^{0}=C_{1}(1-\alpha), C_{1}=\frac{7}{900}+\frac{1}{15} \ln 2=0.0773
$$

3. Case $\alpha \gg 1$. In this case

$$
\begin{aligned}
A_{0}^{0}= & -C_{\infty} \alpha^{4} \\
C_{\infty}= & \frac{1}{8}\left\{\int_{0}^{1} \frac{d x}{x}\left(\frac{2 x}{\left(1+x^{2}\right)^{3 / 2}} \frac{1}{\operatorname{arsh}^{3} x}+\frac{6}{1+x^{2}} \frac{1}{\operatorname{arsh}^{4} x}-\frac{6}{x^{4}}+\frac{2}{15}\right)\right. \\
& \left.+\int_{1}^{\infty} \frac{d x}{x}\left(\frac{2 x}{\left(1+x^{2}\right)^{3 / 2}} \frac{1}{\operatorname{arsh}^{3} x}+\frac{6}{1+x^{2}} \frac{1}{\operatorname{arsh}^{4} x}-\frac{6}{x^{4}}\right)\right\}=0.0173
\end{aligned}
$$

[1] T. W. B. Kibble, J. Phys. A9, 1387 (1976)

[2] A. Vilenkin, Phys. Rep. 121, 263 (1985)

[3] M. Barriola and A. Vilenkin, Phys. Rev. Lett. 63, 341 (1989)

[4] D. Harari and C. Lousto, Phys. Rev. D42, 2626 (1990)

[5] W. A. Hiscock, Phys. Rev. Lett. 64, 344 (1990)

[6] D. P. Bennet and S. H. Rhie, Phys. Rev. Lett. 65, 1709 (1990)

[7] F. D. Mazzitelli and C. O. Lousto, Phys. Rev. D43, 468 (1991) 
[8] W. A. Hiscock, Class. Quantum Grav. 7, L235 (1990)

[9] R. M. Wald, Phys. Rev. D17, 1477 (1978)

[10] H. Ren, Phys. Lett. B325, 149 (1994)

[11] N. D. Birrell and P. C. W. Davies, Quantum Fields in Curved Space, (Cambridge, 1982)

[12] J. D. Bjorken and S. D. Drell, Relativistic Quantum Mechanics, McGraw-Hill, Inc. 1964

[13] I. S. Gradshtein and I. M. Ryzhyk, Table of Integrals, Series and Products, Academic Press, Inc. 1980

[14] R. M. Wald, Commun. Math. Phys. 54, 1 (1977)

[15] S. M. Christensen and M. J. Duff, Phys. Lett. 76B, 571 (1978)

[16] S. F. Adler, J. Liberman and Y. J. Ng, Ann. Phys. 106, 279 (1977)

[17] S. M. Christensen, Phys. Rev. D17, 946 (1978)

[18] V. P. Frolov and E. M. Serebryany, Phys. Rev. D35, 3779 (1987) 